\title{
ANYmal - toward legged robots for harsh environments
}

\section{Journal Article}

\section{Author(s):}

Hutter, Marco (D); Gehring, Christian; Lauber, Andreas; Günther, Fabian; Bellicoso, Carmine D.; Tsounis, Vassilios; Fankhauser, Péter; Diethelm, Remo; Bachmann, Samuel; Blösch, Michael; Kolvenbach, Hendrik; Bjelonic, Marko; Isler, Linus; Meyer, Konrad

Publication date:

2017

\section{Permanent link:}

https://doi.org/10.3929/ethz-b-000194231

\section{Rights / license:}

In Copyright - Non-Commercial Use Permitted

\section{Originally published in:}

Advanced Robotics 31(17), https://doi.org/10.1080/01691864.2017.1378591 


\title{
ANYmal - Toward Legged Robots for Harsh Environments ${ }^{\dagger}$
}

M. Hutter*, C. Gehring, A. Lauber, F. Gunther, C.D. Bellicoso, V. Tsounis, P. Fankhauser, R. Diethelm, S. Bachmann, M. Bloesch, H. Kolvenbach, M. Bjelonic, L. Isler, K. Meyer

\author{
Robotic Systems Lab, ETH Zurich, Switzerland
}

( $v 1.0$ released $X X 2017)$

\begin{abstract}
This paper provides a system overview about ANYmal, a quadrupedal robot developed for operation in harsh environments. The $30 \mathrm{~kg}, 0.5 \mathrm{~m}$ tall robotic dog was built in a modular way for simple maintenance and user-friendly handling, while focusing on high mobility and dynamic motion capability. The system is tightly sealed to reach IP67 standard and protected to survive falls. Rotating lidar sensors in the front and back are used for localization and terrain mapping and compact force sensors in the feet provide accurate measurements about the contact situations. The variable payload, such as a modular pan-tilt head with a variety of inspection sensors, can be exchanged depending on the application. Thanks to novel, compliant joint modules with integrated electronics, ANYmal is precisely torque controllable and very robust against impulsive loads during running or jumping. In a series experiments we demonstrate that ANYmal can execute various climbing maneuvers, walking gaits, as well as a dynamic trot and jump. As special feature, the joints can be fully rotated to switch between X-and O-type kinematic configurations. Detailed measurements unveil a low energy consumption of $280 \mathrm{~W}$ during locomotion, which results in an autonomy of more than $2 \mathrm{~h}$.
\end{abstract}

Keywords: Legged Robot, Quadruped Robot, Field Robotics, Series Elastic Actuation, Autonomous Navigation

\footnotetext{
$\dagger$ This paper is an expanded version of the paper selected from those which were presented at IROS 2016.
}

*Corresponding author. Email: mahutter@ethz.ch 


\section{Introduction}

Legged robots have potential advantages in terms of mobility and versatility compared to tracked or wheeled vehicles. So far, the high technological complexity to build and control such robots has prevented them from being applied in real world scenarios and only few teams managed to develop machines that work beyond laboratory test-bench settings. With major advances over the recent years and pushed by various large-scale research programs and investment from industry, our community is about to overcome the last technical hurdles and make legged robots available for real world applications. Most prominently, the DARPA Robotics Challenge (DRC) brought together some of the best research groups in the field of humanoid robots to successfully use such machines in a disaster mitigation scenario [1. Since the scenario is very close to reality, all teams were forced to massively invest in hardware development to improve not only versatility but also reliability and ruggedness of the robots. These developments resulted in many high-performance machines like ATLAS 2, Valkyrie [3], DRC Hubo [4], HRP2+ [5], Walkman [6] and others, most of them based on earlier robot versions. This new generation of humanoid robots features some sort of force or torque control - either by integrated load cells in the joints or at the endeffector, by pressure transducers in the cylinders, or by a series elasticity in every actuator. This allows them to properly control interaction forces with the environment and hence to balance the system or manipulate objects.

Despite these advances, the locomotory performance of the human-like robots is still far behind the natural counterparts. All these robots are relatively slow, require a lot of power, and can only negotiate small terrain obstacles. Moreover, they are still prototypes that are commonly operated in laboratory environment.

Better locomotion performance in terms of speed, energetic efficiency, and obstacle negotiation skills, is achieved with multi-legged systems. Paramount example is Boston Dynamics' Spot robot, a direct successor of Big Dog [7, as well as its smaller electrically actuated brother Spot-mini. Unfortunately, for both systems there exist no scientific publications. Sim- ilar locomotion performance, demonstrated in various highly dynamic gaits and maneuvers, was also achieved by research groups around IIT's hydraulic quadruped HyQ [8] and its follower HyQ2max [9], MIT's directly electrically actuated cheetah [10], or ETH's serial elastic robot StarlETH [11. These quadrupeds have demonstrated locomotion on different grounds and over substantial obstacles - however, except for some military test applications of the Boston Dynamics' robots (e.g. [12]) none of these machines has been used in a real world application.

The present paper introduces ANYmal (Figure 1), a highly mobile and rugged quadrupedal platform developed for autonomous operation in challenging environments. ANYmal was designed in a modular way to simplify system setup and maintenance. It combines outstanding mobility with dynamic motion capability that enables it to climb large obstacles as well as to dynamically run and jump. This completely autonomous machine is a step towards first real world applications. It is used for search and rescue applications ${ }^{1}$ and industrial inspection $^{2}$ - scenarios with very harsh and demanding environments. In the following, we provide an overview on the underlying mechanics and actuation concept, illustrate the electronics and software setup, sketch out the applied locomotion control algorithms with appropriate references to their implementation, and finally summarize the paper with a series of experiment $\mathrm{s}^{3}$ highlighting the overall system performance.

\section{System description}

ANYmal was specifically built for long endurance autonomous operation in harsh environments. Focus was put on large mobility, fast and dynamic locomotion skills, high robustness, simple maintenance, and safe handling by a single operator. 

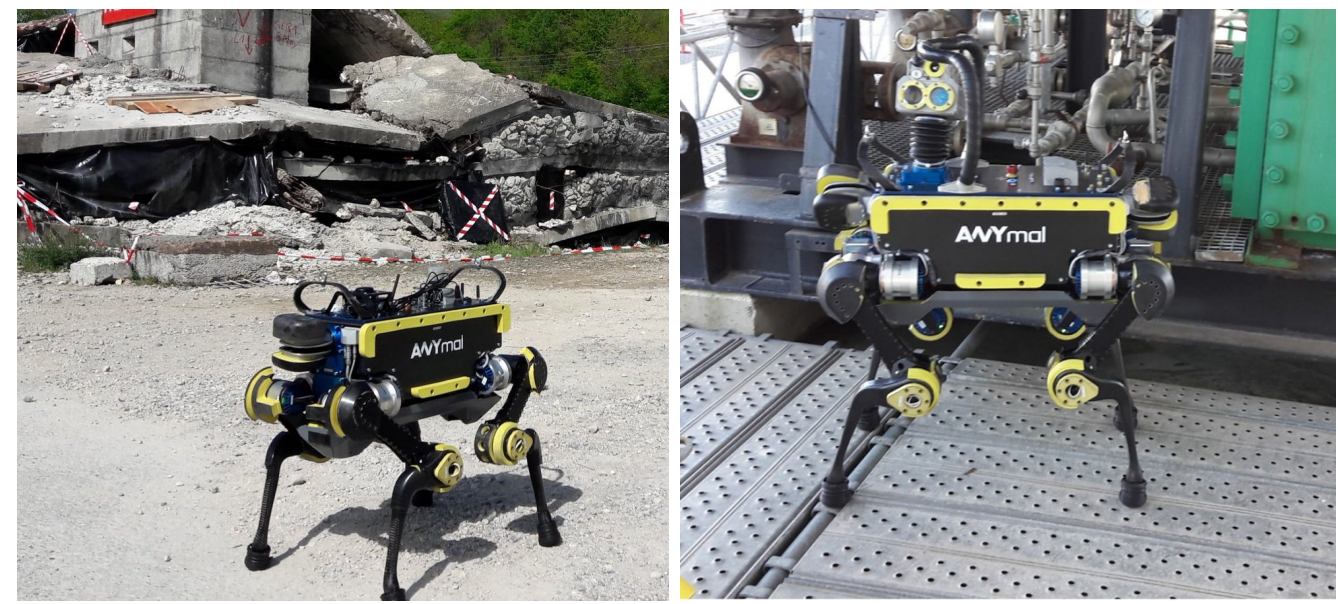

Figure 1. ANYmal, an autonomous quadrupedal robot for rough terrain operation. Depicted are two active scenarios, namely search and rescue (left) as well as industrial inspection (right).

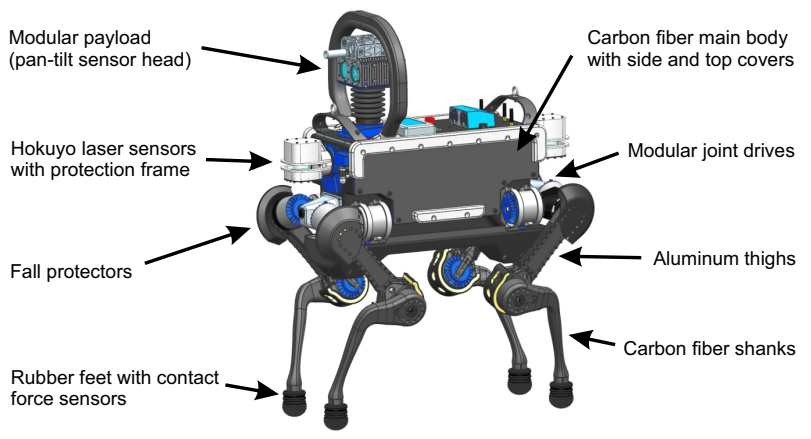

Figure 2. System overview of ANYmal with the ARGOSspecific pan-tilt sensor head for inspection.

\subsection{Overview}

The presented quadrupedal robot, with the main components depicted in Figure 2, features three actuated joints per leg with point feet. It is constructed modularly from a single carbon fiber main body and twelve identical joint units connected by simple mechanical links. Individual joint modules, links, or complete legs can be exchanged in minutes with few screws, which ensures simple and quick maintenance of the system. With an approximate link length of $0.3 \mathrm{~m}$ for thigh and shank, and a total weight of about $30 \mathrm{~kg}$, ANYmal resembles a medium-sized dog. To achieve this lightweight design, the main body and shanks are built from carbon fiber. For better thermal dissipation, the thighs are made

\footnotetext{
${ }^{1}$ http://www.nccr-robotics.ch/RescueRobots

${ }^{2}$ http://www.argos-challenge.com

${ }^{3}$ for illustration, see http://www.rsl.ethz.ch/robotsmedia/anymal
}

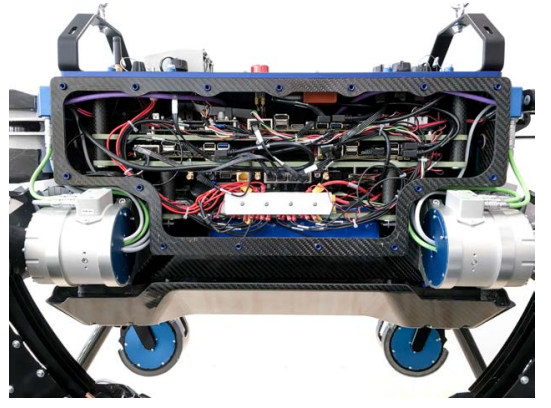

Figure 3. All main-body electronic components are stacked at the top lid. Thanks to two side covers, final cabling and debugging can be conducted while the body is open. Batteries are stored at the lowest point of the main body to keep the center of gravity low.

of aluminum and are thermally coupled to the knee motors. The main body is constructed as a single box that features a mounting interface on the top lid for an application-specific payload such as a pan-tilt inspection sensor head. Force sensors integrated in rubber feet provide haptic information about the contact situation. Two lidar sensors and wide angle cameras are used for environment perception.

\subsubsection{Main body package}

Computers, batteries, network devices, the power management system, and basic navigation sensors are integrated in a single boxshaped and ingress protected main body made of carbon fiber material. In order to combine high stiffness, high robustness, and accessibility of all integrated components, the box features removable covers on both sides and on the 
top (Figure 3).Three fit-PCs, one for locomotion control, one for navigation and motion planning, and a third one dedicated to the specific application, form the powerful brain of ANYmal. They are connected to a gigabit wireless router, which is accessible from an external operator PC. To get proper heat dissipation despite the tightly sealed main body, all electronic components are thermally connected through heatpipes to an external, actively cooled heat sink. The custom Robot Power and System Management (RPSM) consists of an intelligent main board, individual power boards for the legs, and a battery management system connected over CAN bus. This setup is responsible for power distribution among the PCs, sensors, and actuators, for system monitoring and safety, as well as for battery charging and balancing. It is connected to the low-level computer and an external touch screen display, which allows to individually enable $\mathrm{PCs}$, sensors, etc. Two rotating Hokuyo UTM-30LX-EW lidar sensors with fall protection housing and an Xsens MTi-100 IMU are installed for localization and environment mapping. Additional wide-angle cameras in the front and back are available primarily for tele-operation as well as to provide color information in addition to the laser point cloud. As protective element against sharp-pointed objects, ANYmal features a Kevlar plate at the belly. Falls to the side are absorbed by protective memory-shape foam. By these means, the robot can survive falls from more than $0.5 \mathrm{~m}$ height. Since the body is constructed as a single box, it can be pressurized with Nitrogen in order to reach ATEX standard for works in explosive environments.

\subsubsection{Docking station}

ANYmal can autonomously connect to a docking station for recharging the battery and filling the body with inert gas (see Figure 4). Using a special maneuver, ANYmal places the body at the appropriate location on the docking station. The docking station detects this by an inductive sensor and initiates the connection procedure. Thereby, a hatch opens on the docking station and a conically shaped plug is linearly pressed on ANYmal. Two contact pins let the docking station know that docking was completed. This

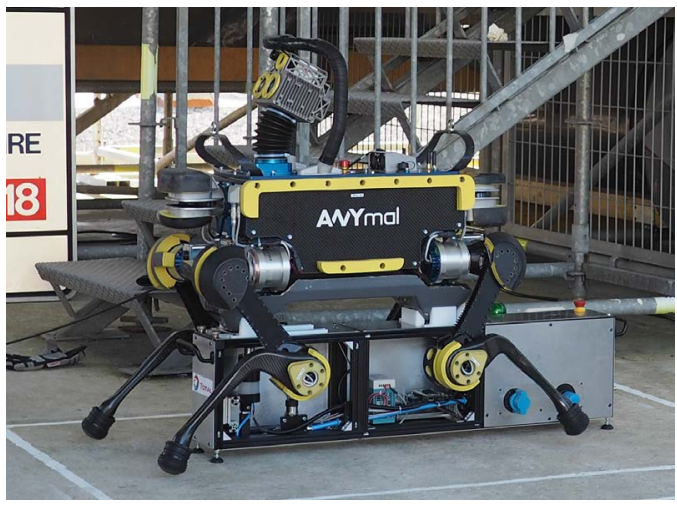

(a) ANYmal seated on docking station

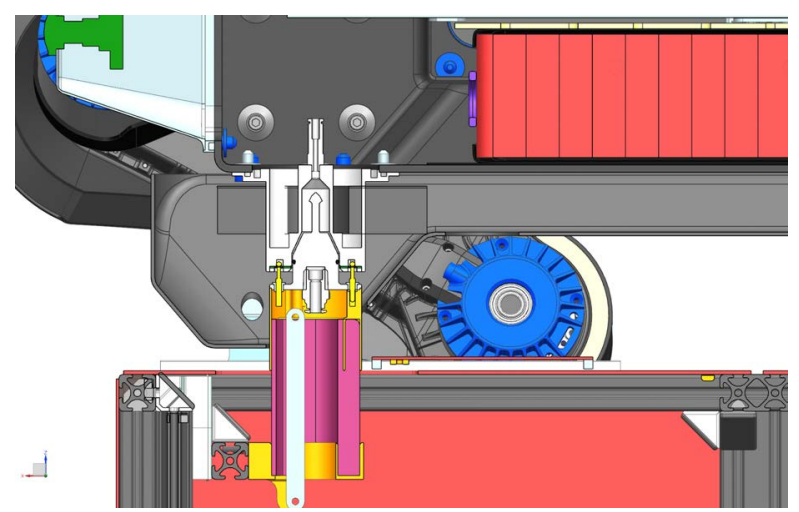

(b) Plug and socket mechanism

Figure 4. The docking station allows for autonomous battery and gas recharging.

automatically opens relays on both sides in order to safely charge the battery while avoiding sparking. In addition to battery charging, the customized plug allows to purge ANYmal with inert gas as required for ATEX certification. After detection of successful docking, Nitrogen is blown through the inlet valve in the center of the plug. An over-pressure outlet valve on the robot ensures that remaining air can escape and that the overall pressure does not exceed 1.2 bar. The pressurization and charging level can be supervised remotely at the operator machine and signal lights indicate the charging status. The docking station automatically returns to the initial configuration after successful charging or in case of interruption.

\subsubsection{Application payload}

Depending on the application, different payloads can be mounted on top of the robot. Potential payloads range from simple sensors to complex 


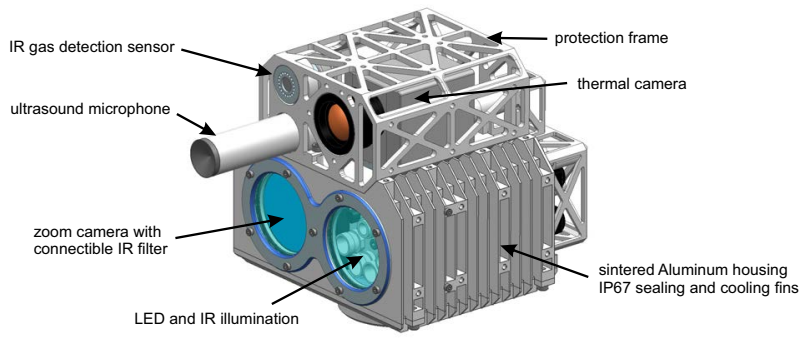

Figure 5. The application payload used during the ARGOS challenge consists of a number of inspection sensors incorporated in a structurally optimized sintered aluminum part.

robotic arms for manipulation. In the example of the ARGOS challenge, a pan-tilt head with various inspection sensors was realized (Figure 5). It includes an optical zoom and thermal camera for visual inspection, a point IR gas detection sensor, an ultrasound microphone for gas leak detection, a regular microphone for sound identification, as well as artificial LED lighting. The sensors and actuators of the application payload can be connected to the application PC in the main body. Furthermore, various voltage levels are provided as DC power source by the RPSM.

\subsubsection{Modular joint setup}

A key to simultaneously achieving the design goals are the robotic joint units described in detail in Section 3. This enabled the creation of a simple mechanical topology with three equal joint units per leg that are linked by rigid mechanical segments and interconnected with a power and communication bus. Since the encapsulated and sealed joint units also include drive electronics, sensing, and the joint axle bearing, the robot does not require any additional bearings, transmission, position encoders, or electronics in its legs. Such a setup combines several advantages: Given the drive units, the robot is simple to manufacture, assemble, and maintain. In case of failure, a complete joint can be quickly exchanged. Furthermore, design variations to build different robots requires only to change the mechanical links.

The joint arrangement of ANYmal is chosen mammalian with successive hip abduction/adduction (HAA), hip flexion/extension (HFE), and knee flexion/extension (KFE). In contrast to its predecessor StarlETH [11], the MIT cheetah [10], IIT HyQ [8], Big Dog [7,

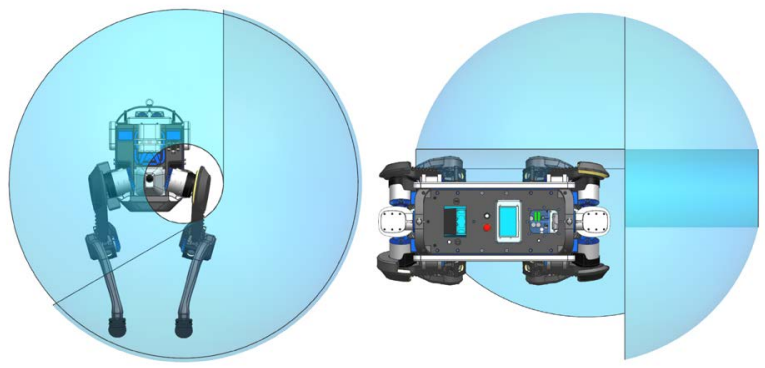

Figure 6. ANYmal features a large range of motion. All joints can be fully rotated.

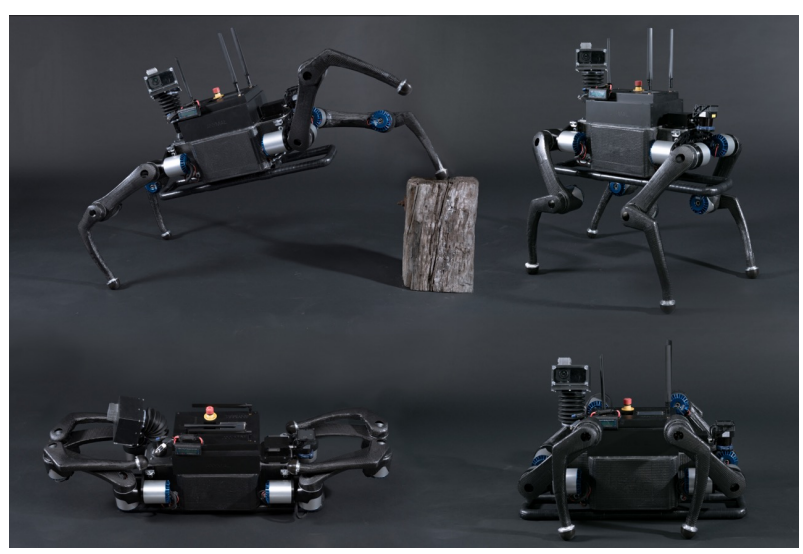

Figure 7. Full rotation in all joints of ANYmal allow for various configurations.

or other legged systems, the leg links of ANYmal are built with an offset such that all joints can be fully rotated. So far, this was typically only done in walking machines like JPL's robosimian [13], which move in a quasi static manner. As depicted in Figure 6, the joint offset enables a huge range of motion which is key to high mobility. With this, ANYmal is able to use its feet high above ground for tasks like opening a door or surmounting large obstacles without shin-hitting problems, it can be folded for transport or deployment, and can change its leg configuration while walking (Figure 7).

\subsection{Software architecture}

The three PCs share the work load for locomotion, navigation, and the application (Figure 8). In the specific application of autonomous inspection in the ARGOS challenge, the third $\mathrm{PC}$ is used for inspection. The data is transferred over the network by the Robot Operating System (ROS) running on a low-latency 


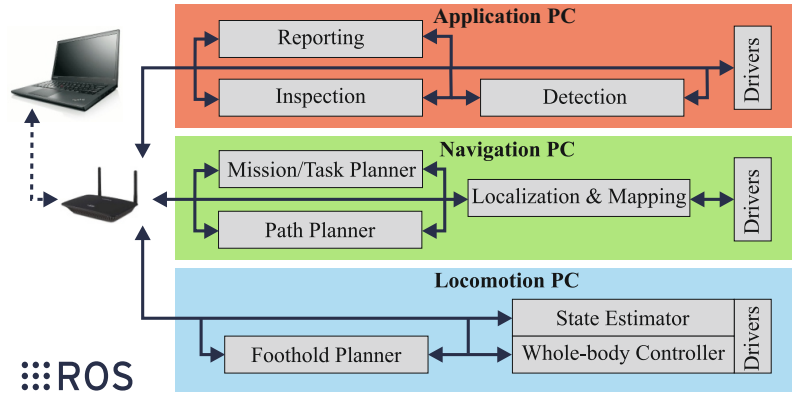

Figure 8. The ROS-based software architecture is implemented on three different PCs.

patched Ubuntu. The ROS master, which manages the connections between the different processes, runs on the locomotion PC. The real-time critical whole-body controller and state estimator are timed by the CAN driver that communicates with the actuator units at $400 \mathrm{~Hz}$. The readings and commands are exchanged through shared memory and published through ROS to less time-critical workers like the foothold planner. The localization and mapping tasks are outsourced to the navigation $\mathrm{PC}$, which is responsible for the laser-based localization and mapping of the environment. High-level navigation tasks are coordinated by a mission planner and executed by a path planner that sends velocity commands to the locomotion controller. The application PC finally is used to handle for example the computationally expensive video processing for inspection or object detection.

\section{ANYdrive - Modular joint units}

Dynamic locomotion imposes very demanding requirements on the actuation system, namely:

- Fast motion tracking

- Low impedance force controllability

- High impact robustness

Furthermore, actuators must be lightweight, compact, and energetically as efficient as possible.

\subsection{A brief review on existing actuation concepts}

The classical approach of electric motors with high-reduction mechanical gears as employed in almost all industrial robot arms does not sat- isfy the second and third requirement. Legged robots using such actuation are limited to slow and static locomotion in order to prevent the actuators from impulsive forces (see e.g. [13]). For dynamic locomotion, three major concepts have been established as adequate actuation technology.

\subsubsection{Hydraulic actuation}

Hydraulic actuators as used in machines like HyQ [8, BigDog [7], or Atlas [2] are naturally robust against impulsive loads and provide high power and force density. Thanks to fast valve units in combination with load cells for force measurement or pressure based force estimation [14, hydraulic actuators provide also high performance torque control.

On the negative side, hydraulic systems tend to be energetically inefficient, in particular when operated with constant pressure. For this reason, many systems used in research still rely on off-board oil or power supply. At the cost of increased system complexity, this can be overcome to certain extent by sophisticated pumps and variable pressure levels. Another problem is scalability, which makes hydraulic legged systems rather large and heavy.

\subsubsection{Pseudo-direct-drive systems}

When using gearing systems of very low reduction and high efficiency, electric actuators can become very transparent and the reflected inertia of the actuation compared to the output remains small. As a result, motor current control, which can be done at very high bandwidth, is equivalent to regulation of the output force [15]. These benefits have been exploited for many years in rehabilitation engineering and for haptic devices. Thanks to recent advances in actuator development, pseudo-direct-drive concepts find application in high-dynamic legged robots as in the example of MIT-cheetah [10, which is able to run and jump at high speeds. Similar concepts were also adopted in the Minitaur project [16], a comparably small and robust quadrupedal device.

Unfortunately, while electric motors have extremely high power, their torque is limited. Therefore, direct actuation without any gear is 


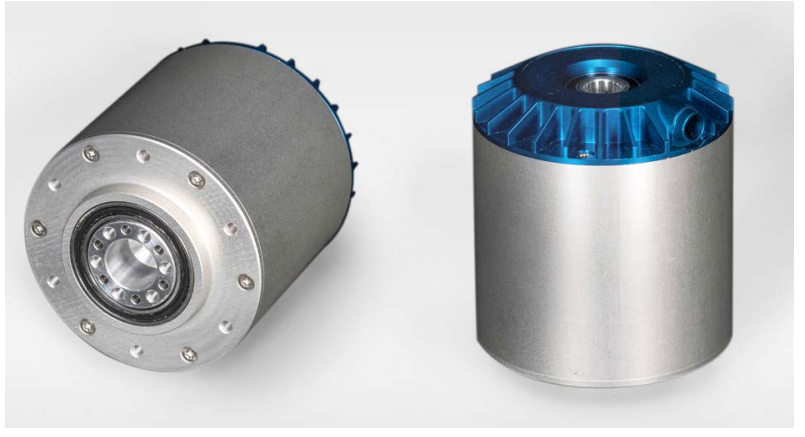

Figure 9. ANYdrive: A compact, series elastic joint units with integrated electronics for advanced interaction.

not possible with existing technology. Furthermore, motors of large diameter must be used to create high forces, which limits flexibility in system design.

\subsubsection{Series elastic actuation}

Inspired from biology and along the seminal work of Pratt [17, the third common actuation approach for legged robots is series elastic actuators. By integrating a carefully selected mechanical compliance between the gearbox output and joint, classical geared motors can be adapted for applications with dynamic interactions. Several state of the art robots like the humanoid Valkyrie 18 or the quadruped StarIETH 11 showed how to use such actuators not only for precise output force regulation, but even to temporarily store energy during locomotion and hence to increase locomotion efficiency [19]. In order to simplify the use of such actuators, different groups target the development of modular units [20, 21].

The mechanical compliance in the system is not only a low pass filter (and hence protection) for the impact loads at the output, but also limits control bandwidth and hence requires careful design of the joint level control structure.

\subsection{ANYdrive overview}

ANYdrive (Figure 9), the joint unit of ANYmal, is a highly integrated series elastic actuator [22]. It is built upon high torque motors and harmonic drive gears in series with a rotational spring. Joint output position and spring deflection are measured using absolute position sensors providing a position accuracy of $0.025^{\circ}$ and

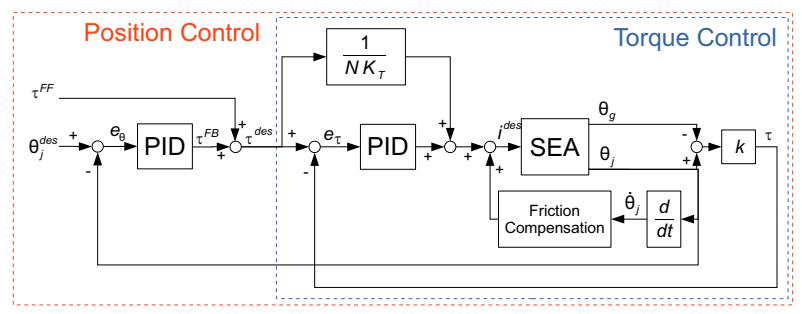

Figure 10. Block diagram of the cascaded joint position and torque control loop. The SEA block represents the physical actuator unit including field oriented control (FOC) to apply the desired current $i^{\text {des }}$.

a torque resolution of $0.08 \mathrm{Nm}$. Thanks to integrated custom motor control electronics, the joint torque, position, and impedance can be directly regulated without any additional components. The corresponding command values are sent over CAN bus using CANopen standard in the first version of the drives and over EtherCAT in the second generation. With a nominal voltage of $48 \mathrm{~V}$, the joint reaches a speed of $12 \mathrm{rad} / \mathrm{s}$ and a maximum torque of approximately $40 \mathrm{Nm}$.

\subsection{Control structure}

Joint torque, position and impedance control is realized as a cascaded structure that considers the motor as torque source (c.f. [23]) as illustrated in Figure 10. Similarly to the work by Paine [24], which is also the basis of the control of Valkyrie [18], we realized a PID torque feedback loop with feedback friction compensation. The position PID control builds upon the torque controller as an additional cascade.

The torque controller tracks a desired torque $\tau^{\text {des }}$ by measuring the actual output torque $\tau$ and by setting the desired current $i^{\text {des }}$ accordingly. The joint torque $\tau$ is calculated from the spring deflection (joint position $\theta_{j}$ minus gear position $\theta_{g}$ ) and the spring constant $k$. The torque controller consists of three elements, i.e. a PID controller, a feedforward term, and a feedback friction compensation. The feedforward term is determined from the inverse of the gear ratio $N$ and the motor constant $K_{T}$, both provided by data sheets. The friction compensation

$$
i_{\text {comp }}\left(\dot{\theta}_{j}\right)=i_{b a} \cdot \operatorname{sighn}\left(\dot{\theta}_{j}, \dot{\theta}_{b a n d}\right)+\mu \dot{\theta}_{j}
$$

takes two effects into account, namely stiction 
and viscous friction. Firstly, the break-away current $i_{b a}$ is modeled as Coulomb friction. To prevent undesired switching around the zero velocity point, it is implemented as simple smooth sign function

$$
\begin{aligned}
& \operatorname{sign}\left(x, x_{b}\right)= \\
& = \begin{cases}-1, & \text { if } x<x_{b} \\
1, & \text { if } x>x_{b} \\
-1+2\left(\frac{x+x_{b}}{2 x_{b}}\right)^{2}\left(2-\frac{x}{x_{b}}\right), & \text { otherwise. }\end{cases}
\end{aligned}
$$

Secondly, the joint velocity dependent viscous friction is linearly modeled with the friction coefficient $\mu$. All these parameters can be experimentally identified from few measurements.

The position controller is a PID controller that tracks a desired joint position $\theta_{j}^{\text {des }}$ by setting a desired torque $\tau^{\text {des }}$. Additional feedforward torque $\tau^{F F}$ is applied to compensate for the configuration depending gravity. For joint impedance regulation, the PD position feedback gains can be tuned as physical parameters to adjust the virtual joint stiffness and damping.

\subsection{Performance evaluation}

The performance of ANYdrive with respect to torque and position reference tracking as well as impulsive disturbance rejection is evaluated on a single axis test bench.

As illustrated in Figure 11(a), the bandwidth for low amplitudes is as high as $70 \mathrm{~Hz}$. Due to motor saturation effects, the bandwidth gradually decreases to $24 \mathrm{~Hz}$ for $10 \mathrm{Nm}$ amplitude. These performance values are substantially higher than what was achieved with our previous system [25] and about the same as in Valkyrie [18. Interestingly, this high performance was achievable without a disturbance observer as used in [26]. One of the main reasons for the good performance of the ANYdrive system lies in the tightly integrated motor electronics which enables high loop rates $(4 \mathrm{kHz})$ at almost no signal delay.

As illustrated in Figure 11(b), the controller is sows a $90 \%$ settling time of $13 \mathrm{~ms}$ for a step of $10 \mathrm{Nm}$ and $35 \mathrm{~ms}$ for a step of $40 \mathrm{Nm}$ with only small overshoot.

Disturbance rejection to impulsive loads is

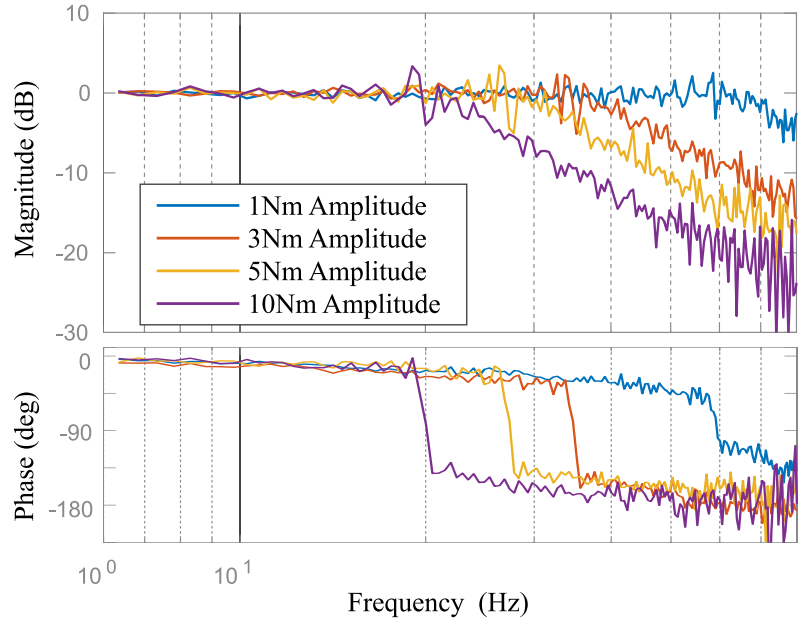

(a) Frequnce response

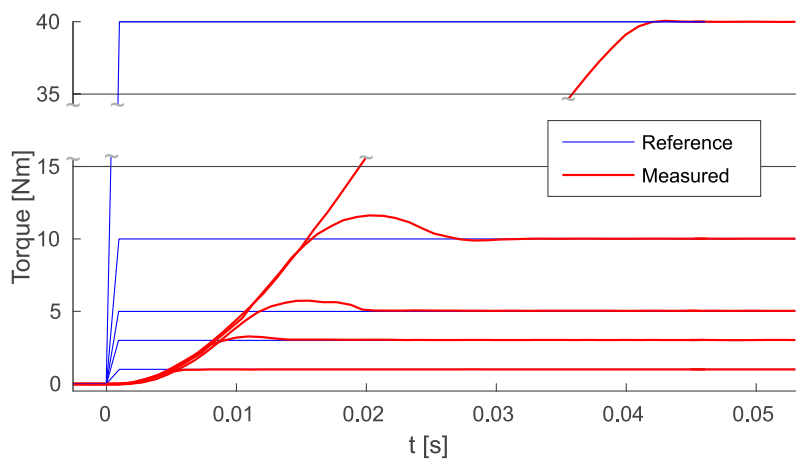

(b) Step answer

Figure 11. a) The experimentally identified torque control transfer function indicates a bandwidth of $70 \mathrm{~Hz}$ for low amplitudes. b) The torque step responses show a quick response time and low overshoot.

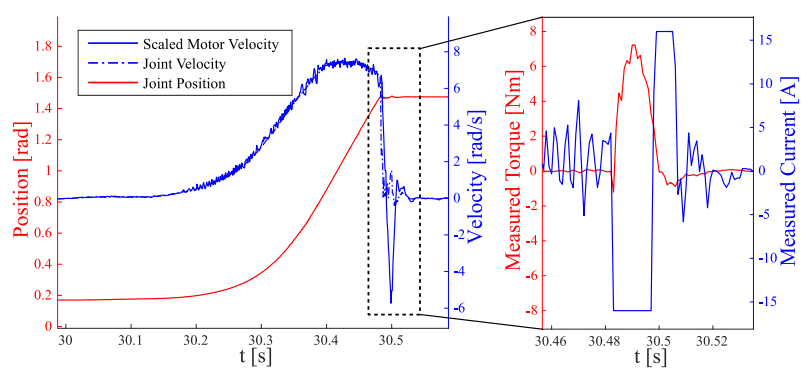

Figure 12. Joint torque during impulsive collision. The motor velocity is scaled with the gear ratio for plotting purposes.

evaluated in a collision test. To this end, a metal pendulum is mounted at the output and the actuator is requested to produce zero torque. The free swinging pendulum is then crashed with high velocity into a hard wall and brought to instantaneous rest (ideal plastic collision with a restitution coefficient of zero). Despite high motor speed before the collision, the gearbox 


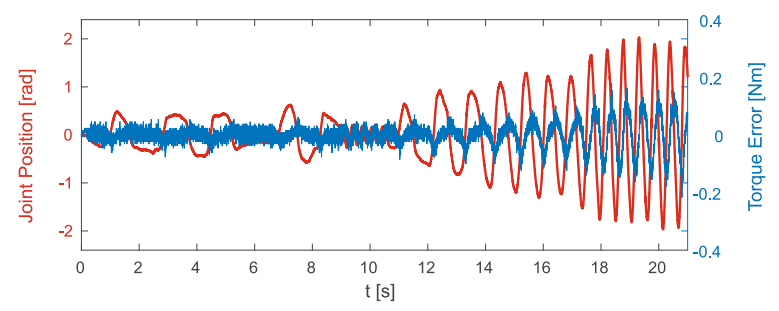

Figure 13. Zero torque tracking error (blue) when the output joint is randomly moved by hand (red).

encounters only little peak torque during the impact (Figure 12). In fact, already $2 \mathrm{~ms}$ after the collision, the motor maximally decelerates to keep the torque in the spring as small as possible. Due to the motor and gearbox inertia, it takes about $10 \mathrm{~ms}$ to bring the motor to a complete rest. If the pendulum collides with the maximum motor velocity, the peak force is smaller than $7 \mathrm{Nm}$. This implies that forces occurring at the gear never exceed the peak loads it is rated for. Hence, the drive is "perfectly robust" against self inflicted collisions.

To evaluate the disturbance rejection, the actuator was commanded to produce zero torque while the output is randomly moved by hand (Figure 13). Despite substantial disturbances (2 rad amplitude and about $4 \mathrm{~Hz}$ motion), the output torque can be kept at less than $0.2 \mathrm{Nm}$. A qualitative comparison to results published for Valkyrie [3] indicates a significantly better disturbance rejection performance.

\section{Locomotion control}

Locomotion control of ANYmal can be roughly split into five key components, namely stateestimation, localization and mapping, navigation, foothold and motion planning, as well as whole-body control.

\subsection{State-estimation, localization, and mapping}

For state-estimation we are building upon the framework described in [27]. Thereby, inertial measurements are tightly fused with kinematic sensory information, which allows to accurately predict attitudes and linear velocities. Moreover, in order to compensate for drift in position and yaw, an iterative closest point (ICP) method [28] is used with the laser data acquired by the two rotating Hokuyos. This setup provides not only information to absolutely localize the robot but additionally gives access to a map of the environment that can be used for navigation as well as for foothold and motion planning.

\subsection{Navigation}

For navigation, the given or acquired map of the environment is transformed into a traversability map using costs like slope, roughness, and step height [29]. Using this map, the sampling-based planner employs the RRT* algorithm to optimize path length and safety. Thereby, we build upon a hierarchical architecture to frequently replan the path during execution as new terrain is perceived with onboard sensors. At this level, only complete footprints are considered instead of individual footholds.

\subsection{Foothold and motion planning}

In the current control setup, we distinguish between dynamic and static locomotion during foothold planning.

In static gaits, individual footholds can be selected purely based on the system kinematics, desired walking speed, as well as the estimated ground elevation from the lidar sensors [30]. This enables various complex maneuvers as required for example to climb obstacles. We worked out a tool [31] for intuitive scripting of various wholebody motions and as an interface for motion planners. Internally, an optimization-based pose finder and spline interpolation is used to generate smooth set points for the whole-body controllers (Section 4.4).

In dynamics gaits, a stepping offset is additionally superimposed in order to balance the machine. Thereby, we make use of a simplified inverted pendulum model [32] (similar to the capture point method [33]) in order to balance disturbances from the nominal motion. Implementation details including a detailed elaboration about the different coordinate frames can be found in 34. 

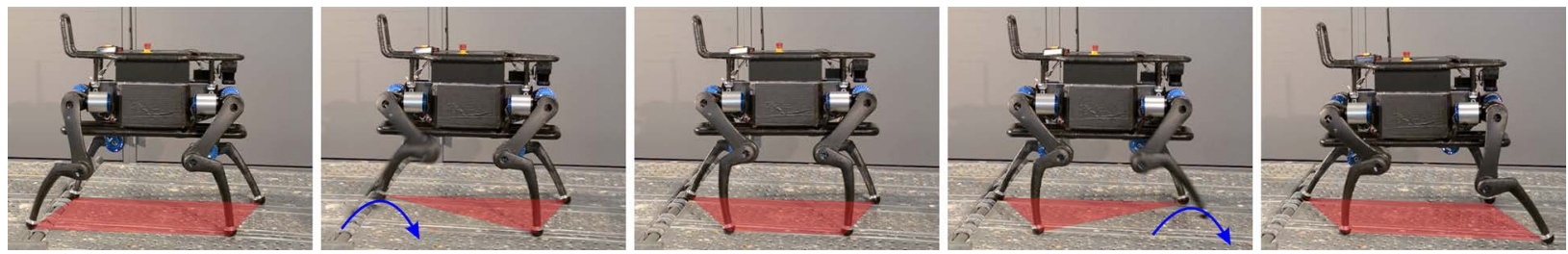

Figure 14. ANYmal can walk by moving one leg at the time and keeping the center of pressure within the support triangle.

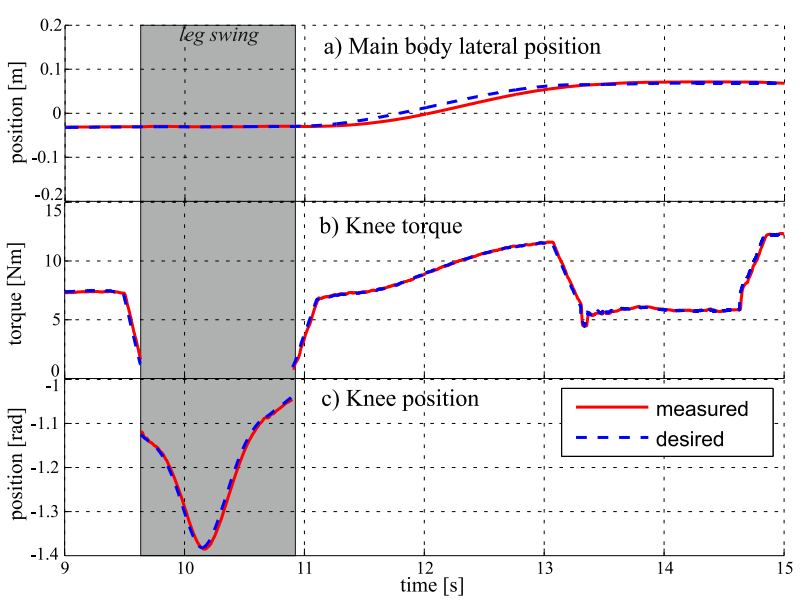

Figure 15. The proposed whole-body control approach results in accurate tracking of the main body position (a), the joint torque (b), and joint position (c).

\subsection{Whole-body control}

Since ANYmal is fully torque controllable, we use model-based control algorithms to optimally distribute ground reaction forces and joint torques. Thereby, locomotion is formulated as an optimization problem with a number of kinematic and dynamic objectives which guarantee that the motion is optimally executed while various constraints are satisfied [35-37]. The applied control accounts for the full system dynamics (prioritized inverse dynamics) or a quasistatic approximation with gravity compensation (extended virtual model control). For both approaches, the optimal actuator commands are found at every time step by solving a constrained optimization problem of prioritized tasks and constraints on joint torques, contact forces, and body motion while ensuring dynamic consistency. An example is that the robot wants to track a desired center of gravity motion and body orientation, while ensuring that the contact forces lie within the friction cones and that all kinds of position and torque limitations are respected.

\section{Experiments}

The performance of ANYmal was tested in different maneuvers and locomotion experiment:4 In order to ensure fast and stable locomotion, particular attention was paid to accurate swing leg position and stance leg force tracking, as well as to a good following behavior of the target base motion.

\subsection{Walking}

ANYmal is able to perform a smooth walking gait, whereby a single leg is moved at the time and the base is shifted in order to maintain balance (Figure 14). As illustrated in Figure 15. joint torques and positions are followed very accurately during the entire gait cycle and hence also the base position can be accurately moved according to the pre-planned trajectory. It is important to know that these results were achieved without any joint level position or impedance regulation. By applying a classical ZMP planner [38], forward locomotion results in a smooth and almost straight line of the base with an approximate velocity of $0.3 \mathrm{~m} / \mathrm{s}$. Thanks to the full rotation capability, the motion planner does not have to account for complex geometric collision constraints but only for limited abduction/adduction freedom of the hip joint due to the main body. As we can see from the snapshots (Figure 14), ANYmal can take fairly big steps.

\section{2. $\quad$ Trotting}

ANYmal is able to trot on different grounds and under substantial external disturbances like unperceived obstacles on the ground or external pushes to the main body. Similar to the walk-

${ }^{4}$ For videos, see http://www.rsl.ethz.ch/robots-media/anymal 

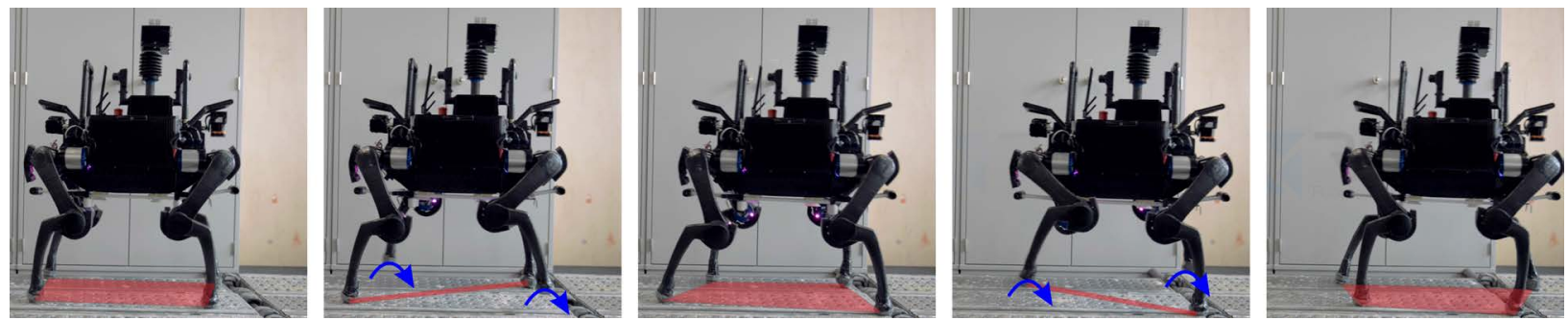

Figure 16. In most situations and for most terrains, ANYmal applies a walking trot with a duty cycle of $50 \%$, whereby the two diagonal legs are moved together.
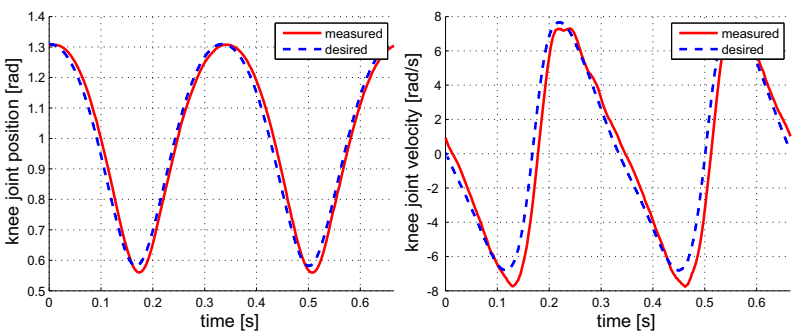

Figure 17. Tracking performance of the position and velocity of the knee joint.

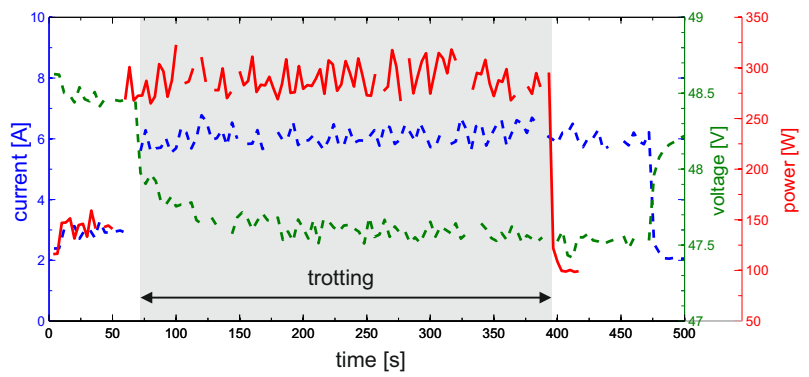

Figure 18. Power consumption during trotting.

ing gait, already the first experiments unveiled large advantages of the big range of motion as the legs can be moved relatively far in all directions. Using a $50 \%$ duty cycle gait, the machine achieves a speed of about $0.8 \mathrm{~m} / \mathrm{s}$. Thereby, joint positions and velocities are followed accurately despite the joint compliance (Figure 17).

A thorough evaluation of the overall energy consumption at the onboard battery indicates a high locomotion efficiency. As depicted in Figure 18, ANYmal requires in average about $290 \mathrm{~W}$ with about $5 \%$ fluctuation when trotting, about $100 \mathrm{~W}$ is consumed while idling in standing configuration. The total power consumption corresponds to a cost of transport of about 1.2. These measurements are comparable to our previous results with StarlETH [39]. With its current onboard battery, the machine can operate autonomously for more than $2 \mathrm{~h}$.

\section{3. $\quad$ Stairs and steps}

ANYmal can apply a standard walking gait to move across steps or stairs as they occur in our daily environment (Figures 19(a) and 19(c) and ??). Depending on the step height, the body and leg motion is simultaneously optimized in order to prevent any collision with the environment. In case of reaching limits, potential shin hitting problems or tight spaces, the leg can be fully turned to change from $\mathrm{X}$ - to Oconfiguration (Figure 19(d)).

\subsection{Stair Crawling}

As a proof of high mobility, ANYmal was further tested for the ability to get up an industrial ladder of more than $50^{\circ}$ inclination. To do this in a save manner and to prevent falling by all possible means, a turtle like crawling gait was implemented. The main body lies on the ground, the legs are moved to find the next stable contact holds, and the machine is subsequently pulled upwards (see Figure 20). Due to ANYmal's large range of motion, the legs can be turned overhead to prevent collision with the ground or side rails. For this maneuver, a joint impedance control mode with gravity compensation was implemented in order to be robust against difficult contact situations.

\subsection{Jumping}

To support the claim of dynamics motion capability, different jumping experiments were conducted. A single maneuver is depicted in Figure 21. In phase A, the body is moved about $10 \mathrm{~cm}$ downwards to prepare for acceleration. In phase B, the base is accelerated upwards by setting the base target position above the nominal 


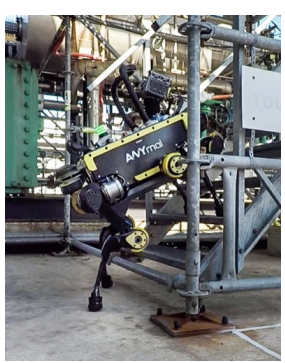

(a) Stair walk

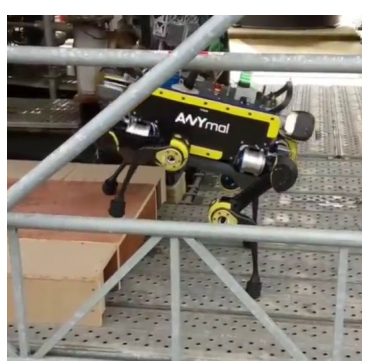

(b) Step walk

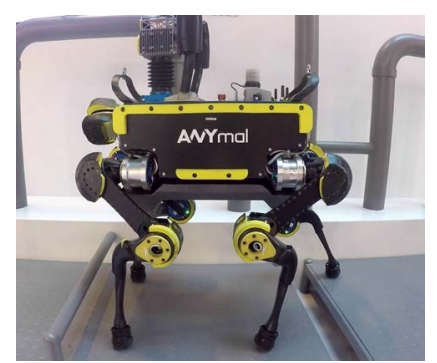

(c) Gaps and hurdle walk

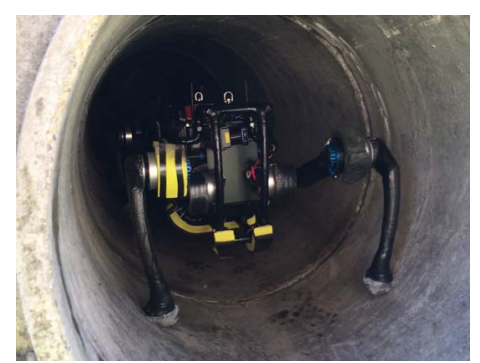

(d) Spider crawling

Figure 19. With its large range of motion, ANYmal can move over obstacles such as steps or stairs, and through narrow spaces 31.

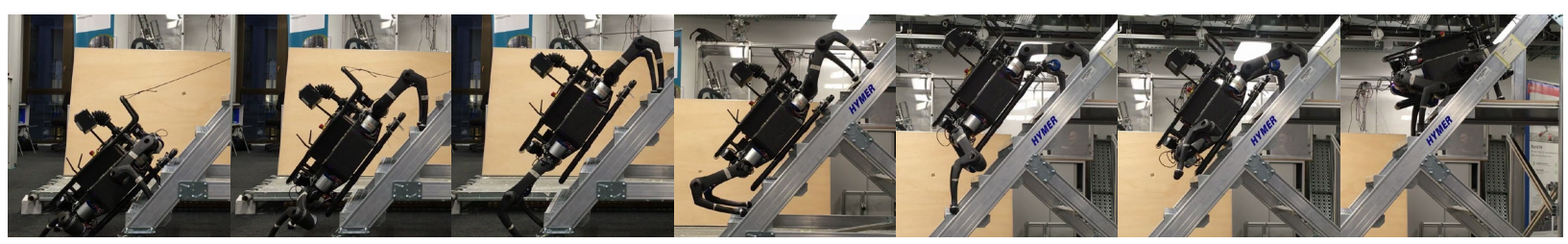

Figure 20. ANYmal can crawl up ladders of more than $50^{\circ}$ inclination. Image taken from 31 .
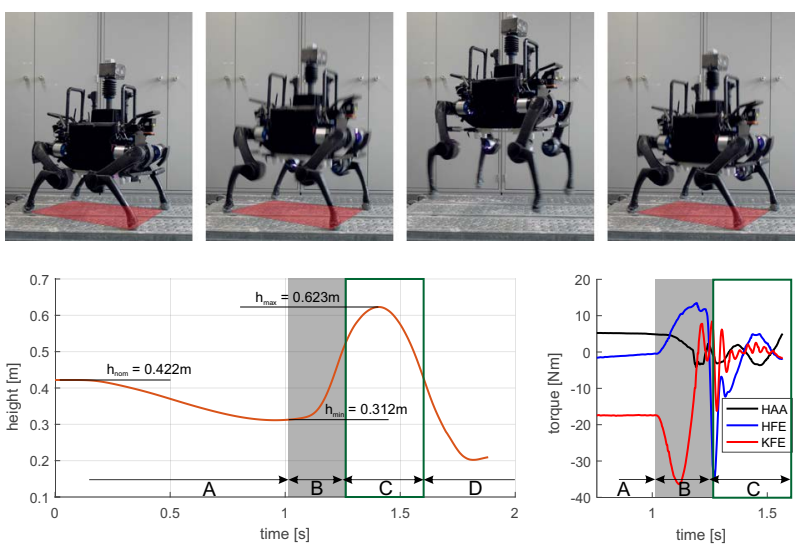

Figure 21. ANYmal can perform simple jumps. The four images correspond to the three phases indicated in the estimated height (left) and joint torques (right) during a single jump: During phase $\mathrm{A}$, the robot moves to a crouched position. In phase $\mathrm{B}$, the body is accelerated upwards, whereby the joint torques in particular in $\mathrm{KFE}$ reach about $36 \mathrm{Nm}$. In flight phase $\mathrm{C}$, which takes about $0.34 \mathrm{~s}$, the body jumps more than $20 \mathrm{~cm}$ high. In phase D finally, the body is decelerated after landing.

height. Since the same controller is running as during walking or trotting, the system is still fully balanced and distributes the accelerating contact forces optimally among the four legs. After lift-off, the legs are brought to a predefined landing configuration before landing. In this phase, no angular momentum control is applied and the robot jumps about $20 \mathrm{~cm}$ high. After landing, the reference center of gravity height is set again to the nominal value such that the downward motion is damped out while the system is balanced. For periodic jumping, the presented procedure can be simply executed in a periodic manner.

\section{Conclusion and future work}

ANYmal is considered a step towards legged robots for harsh environments. It unifies high mobility with dynamic locomotion capability in a robust quadrupedal platform.

From the beginning of the design phase, special attention was paid to make the system rugged and easy to maintain. This was achieved with the joint units ANYdrive that allow to modularly creating robots of different kinematic structure. In case of failure, these modules can be quickly exchanged without special knowhow. They are based on a series elastic concept as already implemented on StarlETH, where we did not have a single gearbox failure in 4 years of almost daily operation with high-dynamic maneuvers. The presented single joint experiments support the claim of robustness since even completely plastic and unexpected output collisions do not lead to higher gearbox loads than during nominal operation.

Beside the highly improved ingress and fall 
protection, the biggest advantage of ANYmal is clearly the outstanding range of motion in all joints. This enables a large variety of maneuvers to overcome obstacles or to get up after falling. Furthermore, it simplifies motion planning as there are less internal system constraints. The initial objectives of creating a dynamic and highly mobile autonomous walking machine could be confirmed in preliminary experiments including different climbing maneuvers, ZMPbased walking, dynamic trotting, and jumping. The present development shall enable deployment of legged robots in real world scenarios such as for search and rescue or industrial inspection.

\section{Funding}

This work was supported in part by the Swiss National Science Foundation (SNF) through the National Centre of Competence in Research Robotics, by the Gebert Ruf Foundation, by Wyss Zurich, and by TOTAL SA through the ARGOS Challenge.

\section{Notes on contributors}

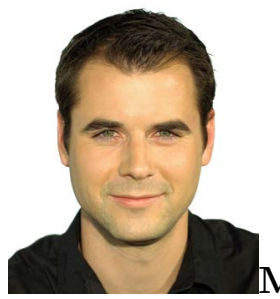

Marco Hutter is Assistant Professor for Robotic Systems at ETH Zurich and Branco Weiss Fellow. He studied mechanical engineering and conducted his doctoral degree in robotics at ETH Zurich. Marco Hutter is part of the National Centre of Competence in Research (NCCR) Robotics and NCCR Digital Fabrication. His research interests are in the development of novel machines and actuation concepts, including the underlying control, planning and optimization algorithms for locomotion and manipulation.

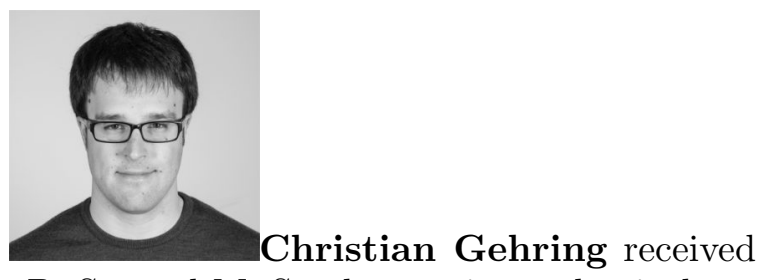

his B. Sc. and M. Sc. degrees in mechanical engineering from ETH Zurich, in 2008 and 2011, respectively, and his doctoral degree from ETH Zurich in 2017. He worked at the Autonomous Systems Lab at ETH Zurich and collaborated with Disney Research Zurich during his studies on motion planning and control for agile quadruped robots. He is co-founder of ANYbotics, which provides solutions for a new level of mobility and interaction capabilities for robots. He currently holds a Postdoctoral researcher position at the Robotic Systems Lab at ETH Zurich and is interested in control of agile and robust quadrupedal locomotion.

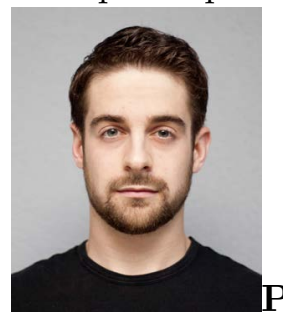

Ph.D. student at ETH ANYbotics. His current work focuses on mapping, control, and motion planning for legged robots in rough terrain. Péter Fankhauser received his B.Sc. degree in mechanical engineering and his M.Sc. degree in Robotics, Systems and Control from ETH Zurich in 2010 and 2012.

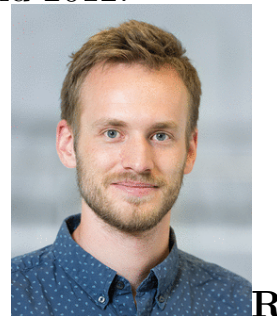
M.Sc. in Mechanical Engineering at ETH Zurich in 2013. During his masters studies, using state of the art contact laws, he programmed a physics simulation that is used for gait learning and control optimization of walking robots. His subsequent work at the Autonomous Systems Lab at ETH Zurich was dedicated to ANYmal's highlevel software development, including localization, mapping, navigation and mission execution 
which was used during the ARGOS challenge.

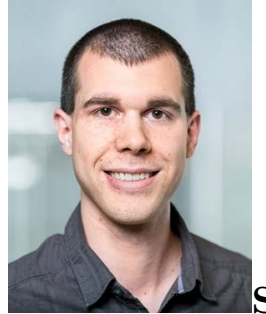

Samuel Bachmann received

his M.Sc. in Robotics, Systems and Control at ETH Zurich in 2015. His master thesis was about the visual inspection of pressure gauges and valve positions for the ARGOS Challenge. Afterwards, he continued his work at the Autonomous Systems Lab, ETH Zurich and was responsible for all the ARGOS visual inspection tasks as well as detecting various sound patterns. He contributed a great part of the robot user interface and is part of the software deployment team.

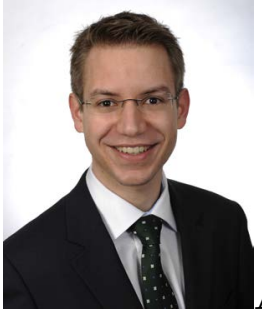

Andreas Lauber received his M.Sc. in Mechanical Engineering at ETH Zurich in 2011 and is a co-founder of ANYbotics. After a successful development project for the European Space Agency and two years working for the swiss engineering consultant Helbling Technik AG, he joined RSL in 2014 as a design engineer. Andreas is dedicated to engineering tasks from mechanical design to production of actuators and whole robotics systems.

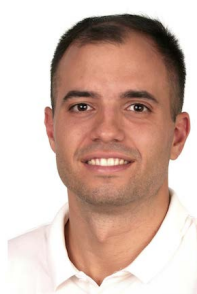

Marko Bjelonic received the B.S. and M.S. degrees in mechanical engineering from Technische Universität Darmstadt, Germany, in 2013 and 2016, respectively. He is currently working toward the Ph.D. degree with the Robotic Systems Lab of ETH Zürich, Switzerland. His research interest includes control and autonomous navigation of legged systems in challenging environments, and the transfer of this technology to robotic and non-robotic ap- plications.

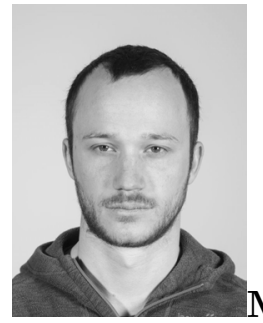

Michael Bloesch received his doctoral degree from ETH Zurich in 2017. He currently holds a Dyson Fellowship at the Dyson Robotics Lab at Imperial College London, where he is working on deep learning supported SLAM methodologies. His research interests include computer vision and sensor fusion frameworks as well as their application to different robotic platforms.

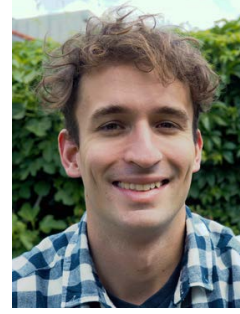
ceived his B.Sc. and M.Sc.in Mechanical Engineering from RWTH Aachen University, in 2013 and 2014, respectively. After a training period at the European Space Agency he joined the Robotic Systems Lab of ETH Zürich in 2016 to persue a Ph.D. degree. His research interests include robotic system design and the application of legged systems for extraterrestrial exploration.

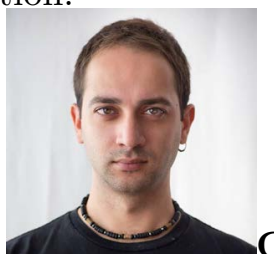

C. Dario Bellicoso received his B.Sc. and M.Sc. in Automation Engineering from the University of Naples Federico II. After an internship as a research assistant at the Prisma Lab in Naples with Prof. Bruno Siciliano he joined the Robotic Systems Lab of ETH Zurich as a Ph.D student. His research focuses on motion planning and whole-body control for legged robots over rough terrain. 


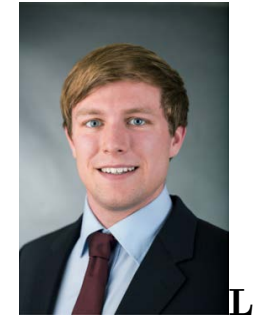

Linus Isler received his M.Sc. in Electrical Engineering at ETH Zurich in 2016. His work as a software engineer for the Robotic Systems Lab of ETH Zürich includes teleoperation, navigation and obstacle negotiation for legged robots.

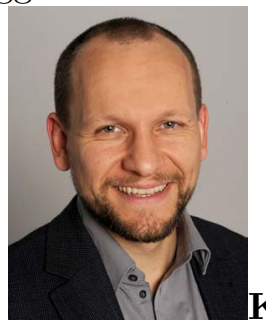
Dipl. Ing. (FH) in Electrical Engineering from the University of Applied Sciences in Konstanz, Germany in 2007. He enhanced his knowledge in engineering services, test automation and industrial production during his work in the industry until 2013. After his self-employment he joined ETH Zürich in 2016. He focuses now on the hardware development in respect to embedded platforms, power electronics and communication for autonomous systems.

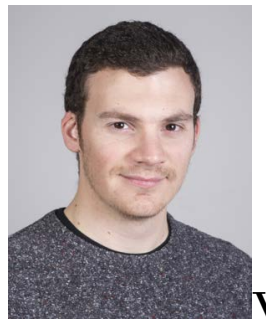
Dipl. Eng. in Electrical and Computer Engineering from the National Technical University of Athens (NTUA) in Athens, Greece in 2014. Having worked as an stagiaire (intern) at the $\mathrm{Au}-$ tomation and Robotics Lab (ARL), at the European Space Agency (ESA) at Noordwijk in The Netherlands, and subsequently as a mechatronics engineer in Athens, he later joined RSL in 2015 as an engineer developing the ANYdrive SEAs. He is currently pursuing doctoral studies at RSL, focusing on learning-based control methodologies for legged locomotion.

\section{References}

[1] Krotkov E, Hackett D, Jackel L, et al. The DARPA Robotics Challenge Finals: Results and Perspectives. Journal of Field Robotics. 2017 mar;34:229-240; Available from: http://doi. wiley.com/10.1002/rob.21683

[2] Boston Dynamics. Atlas robot. Accessed: 2017-01-30; Available from: http://www. bostondynamics.com/robot_Atlas.html.

[3] Radford NA, et al. Valkyrie: NASA's First Bipedal Humanoid Robot. Journal of Field Robotics. 2015;32:397-419; Available from: http://doi.wiley.com/10.1002/rob.21560.

[4] Wang H, Zheng YF, Jun Y, et al. DRC-hubo walking on rough terrains. In: IEEE International Conference on Technologies for Practical Robot Applications (TePRA); apr. IEEE; 2014. p. 1-6; Available from: http://dx.doi. org/10.1109/TePRA.2014.6869151.

[5] Kaneko K, et al. Humanoid robot HRP-2Kai Improvement of HRP-2 towards disaster response tasks. In: 2015 IEEE-RAS 15th International Conference on Humanoid Robots (Humanoids); 2015. p. 132-139; Available from: http://ieeexplore.ieee.org/lpdocs/ epic03/wrapper.htm?arnumber=7363526.

[6] Tsagarakis N, Caldwell D, Bicchi A, et al. WALK-MAN: A High Performance Humanoid Platform for Realistic Environments. Journal of Field Robotics (JFR). 2016;.

[7] Raibert M, Blankespoor K, Nelson G, et al. BigDog, the Rough-Terrain Quadruped Robot. IFAC Proceedings Volumes. 2008;41:1082210825; Available from: http://dx.doi.org/ 10.3182/20080706-5-KR-1001.01833.

[8] Semini C, Tsagarakis NG, Guglielmino E, et al. Design of HyQ - a hydraulically and electrically actuated quadruped robot. Proceedings of the Institution of Mechanical Engineers, Part I: Journal of Systems and Control Engineering. 2011;225:831-849; Available from: http: //dx.doi.orgi/10.1177/0959651811402275.

[9] Semini C, Barasuol V, Boaventura T, et al. Towards versatile legged robots through active impedance control. The International Journal of Robotics Research. 2015;34:10031020; Available from: http://ijr.sagepub. com/cgi/doi/10.1177/0278364915578839.

[10] Seok S, Wang A, Otten D, et al. Design principles for highly efficient quadrupeds and implementation on the MIT Cheetah robot. In: IEEE International Conference on Robotics and $\mathrm{Au}-$ tomation (ICRA); 2013. p. 3307-3312; Available from: http://ieeexplore.ieee.org/lpdocs/ 
epic03/wrapper.htm?arnumber $=6631038$

[11] Hutter M, Gehring C, Bloesch M, et al. StarlETH: a Compliant Quadrupedal Robot for Fast, Efficient, and Versatile Locomotion. In: International Conference on Climbing and Walking Robots (CLAWAR); 2012. p. 483-490; Available from: http://dx.doi.org/10.1142/ 9789814415958_0062.

[12] Defense-Updatecom. Innovative robotic systems supporting Marines during warfighting experiment in Hawaii. 2014; Available from: http://defense-update.com/20140801_1s3_ guss_robots_at_awe.html\#.VG0-u8J0wdU.

[13] Hebert P, et al. Mobile Manipulation and Mobility as Manipulation-Design and Algorithms of RoboSimian. Journal of Field Robotics. 2015;32:255-274; Available from: http://doi. wiley.com/10.1002/rob.21566

[14] Boaventura TC. Hydraulic Compliance Control of the Quadruped Robot HyQ [dissertation]. University of Genoa, Italy and Istituto Italiano di Tecnologia (IIT); 2013.

[15] Seok S, Wang A, Otten D, et al. Actuator design for high force proprioceptive control in fast legged locomotion. In: IEEE/RSJ International Conference on Intelligent Robots and Systems (IROS); 2012. p. 1970-1975; Available from: http://ieeexplore.ieee.org/lpdocs/ epic03/wrapper.htm?arnumber $=6386252$

[16] Kenneally G, De A, Koditschek DE. Design Principles for a Family of Direct-Drive Legged Robots. IEEE Robotics and Automation Letters. 2016 jul;1:900-907; Available from: http://ieeexplore.ieee.org/document/ 7403902/.

[17] Pratt GA, Williamson MM. Series elastic actuators. In: IEEE International Conference on Intelligent Robots and Systems (IROS). MIT; 1995. p. 399-406; Available from: http://dx. doi.org/10.1109/IROS.1995.525827.

[18] Paine N, Mehling JS, Holley J, et al. Actuator Control for the NASA-JSC Valkyrie Humanoid Robot: A Decoupled Dynamics Approach for Torque Control of Series Elastic Robots. Journal of Field Robotics. 2015 may;32:378-396; Available from: http://doi.wiley.com/10.1002/ rob.21556.

[19] Hutter M, Remy CD, Hoepflinger MA, et al. Efficient and Versatile Locomotion with Highly Compliant Legs. IEEE/ASME Transactions on Mechatronics. 2013;18:449-458; Available from: http://dx.doi.org/10.1109/TMECH. 2012 . 2222430

[20] Rollinson D, et al. Design and architecture of a series elastic snake robot. In: IEEE/RSJ Inter- national Conference on Intelligent Robots and Systems (IROS); 2014. p. 4630-4636; Available from: http://ieeexplore.ieee.org/lpdocs/ epic03/wrapper.htm?arnumber=6943219.

[21] Paskarbeit J, Annunziata S, Basa D, et al. A self-contained, elastic joint drive for robotics applications based on a sensorized elastomer couplingDesign and identification. Sensors and Actuators A: Physical. 2013 sep;199:56-66; Available from: http://linkinghub.elsevier. com/retrieve/pii/S0924424713001921.

[22] Hutter M, Bodie K, Lauber A, et al. EP16181251 - Joint unit, joint system, robot for manipulation and/or transportation, robotic exoskeleton system and method for manipulation and/or transportation. 2016.

[23] Pratt GA, Willisson P, Bolton C, et al. Late motor processing in low-impedance robots: impedance control of series-elastic actuators. In: American Control Conference (ACC); Vol. 4; 2004. p. 3245-3251; Available from: http://ieeexplore.ieee.org/xpls/abs_ all.jsp?arnumber=1384410.

[24] Paine NA. High-Performance Series Elastic Actuation [dissertation]. The University of Texas at Austin; 2014.

[25] Hutter M, Remy CD, Hoepflinger MH, et al. High Compliant Series Elastic Actuation for the Robotic Leg ScarlETH. In: International Conference on Climbing and Walking Robots (CLAWAR). Paris, Fr; 2011. p. 507-514; Available from: http://dx.doi.org/10.1142/ 9789814374286_0059.

[26] Paine N, Oh S, Sentis L. Design and Control Considerations for HighPerformance Series Elastic Actuators. IEEE/ASME Transactions on Mechatronics. 2014 jun;19:1080-1091; Available from: http://ieeexplore.ieee.org/lpdocs/ epic03/wrapper.htm?arnumber $=6555856$.

[27] Bloesch M, Gehring C, Fankhauser P, et al. State estimation for legged robots on unstable and slippery terrain. In: IEEE/RSJ International Conference on Intelligent Robots and Systems (IROS); 2013. p. 6058-6064; Available from: http://dx.doi.org/10.1109/ IROS.2013.6697236.

[28] Pomerleau F. Applied registration for robotics [dissertation]. ETH; 2013; Available from: http://e-citations.ethbib.ethz.ch/view/ pub:111721.

[29] Wermelinger M, Fankhauser P, Diethelm R, et al. Navigation planning for legged robots in challenging terrain. In: IEEE/RSJ International Conference on Intelligent Robots and Systems 
(IROS); oct. IEEE; 2016. p. 1184-1189; Available from: https://doi.org/10.1109/IROS. 2016.7759199.

[30] Fankhauser P, Bloesch M, Gehring C, et al. Robot-Centric Elevation Mapping with Uncertainty Estimates. In: International Conference on Climbing and Walking Robots (CLAWAR); 2014. p. 433-440; Available from: http://dx. doi.org/10.1142/9789814623353_0051.

[31] Fankhauser P, Dario Bellicoso C, Gehring C, et al. Free Gait An architecture for the versatile control of legged robots. In: IEEERAS International Conference on Humanoid Robots (Humanoids); nov. IEEE; 2016. p. 10521058; Available from: http://ieeexplore. ieee.org/document/7803401/.

[32] Gehring C, et al. Towards Automatic Discovery of Agile Gaits for Quadrupedal Robots. In: IEEE International Conference on Robotics and Automation (ICRA); 2014. p. 4243-4248; Available from: http://dx.doi.org/10.1109/ ICRA.2014.6907476.

[33] Pratt J, Carff J, Drakunov S, et al. Capture Point: A Step toward Humanoid Push Recovery. In: IEEE-RAS International Conference on $\mathrm{Hu}-$ manoid Robots (Humanoids); 2006. p. 200-207; Available from: http://dx.doi.org/10.1109/ ICHR . 2006.321385.

[34] Gehring C. Planning and Control for Agile Quadruped Robots [dissertation]. ETH Zurich; 2016.

[35] Hutter M, Sommer H, Gehring C, et al. Quadrupedal locomotion using hierarchical operational space control. The International Journal of Robotics Research (IJRR). 2014 may; 33:1062-1077; Available from: http://dx.doi. org/10.1177/0278364913519834

[36] Gehring C, et al. Practice Makes Perfect: An Optimization-Based Approach to Controlling Agile Motions for a Quadruped Robot. IEEE Robotics \& Automation Magazine. 2016;23:34-43; Available from: http://ieeexplore.ieee.org/lpdocs/ epic03/wrapper.htm?arnumber $=7414416$

[37] Bellicoso CD, Gehring C, Hwangbo J, et al. Perception-less terrain adaptation through whole body control and hierarchical optimization. In: IEEE-RAS 16th International Conference on Humanoid Robots (Humanoids); nov. IEEE; 2016. p. 558-564; Available from: http://dx.doi.org/10.1109/HUMANOIDS. 2016.7803330.

[38] Vukobratovic M, Juricic D. Contribution to the Synthesis of Biped Gait. IEEE Transactions on Biomedical Engineering. 1969 jan;BME-16:1-6;
Available from: http://dx.doi.org/10.1109/ TBME. 1969.4502596.

[39] Hutter M, Gehring C, Hopflinger MA, et al. Toward Combining Speed, Efficiency, Versatility, and Robustness in an Autonomous Quadruped. IEEE Transactions on Robotics. 2014 dec; 30:1427-1440; Available from: http://dx.doi . org/10.1109/TRO.2014.2360493 